\title{
ON THE TRANSFORMATION OF THE LINEAR DIFFERENTIAL EQUATION INTO A SYSTEM OF THE FIRST ORDER EQUATIONS
}

\author{
M.I. Ayzatsky \\ National Science Center “Kharkov Institute of Physics and Technology”, Kharkiv, Ukraine \\ E-mail: aizatsky@kipt.kharkov.ua
}

The generalization of the transformation of the linear differential equation into a system of the first order equations is presented. The proposed transformation gives possibility to get new forms of the $N$-dimensional system of first order equations that can be useful for analysis of the solutions of the $N$-th-order differential equations. In particular, for the third-order linear equation the nonlinear second-order equation that plays the same role as the Riccati equation for second-order linear equation is obtained.

PACS: $02.30 . \mathrm{Hq}$

\section{INTRODUCTION} tion

It is well known that the $N$-th order differential equa-

$$
y^{(N)}+f_{N-1}(t) y^{(N-1)}+\ldots \ldots . . f_{1}(t) y^{\prime}+f_{0}(t) y+f(t)=0
$$

can be converted into an $N$-dimensional system of first order equations. There are various reasons for doing this, one being that a first order system is much easier to solve numerically as the most differential equations we encounter in physics, economics and engineering do not have exact solutions.

The most common method is introducing a number of new variables

and representing (1) as

$$
y^{(m)}=y_{m}, \quad m=1,2, \ldots, N-1
$$

$$
Y^{\prime}=M Y+H,
$$

where $H=(0,0 \ldots,-f(t))^{T}, Y=\left(y, y_{1}, \ldots, y_{N-1}\right)^{T}$,

$$
M=\left(\begin{array}{ccccc}
0 & 1 & 0 & \ldots & 0 \\
0 & 0 & 1 & \ldots & 0 \\
\ldots & \ldots & \ldots & \ldots & \ldots \\
0 & 0 & 0 & \ldots & 1 \\
f_{0} & f_{1} & f_{2} & \ldots & f_{N-1}
\end{array}\right) .
$$

This system of equations can be subjected to further transformations (see, for example, [1 - 5]).

For the second order differential equation

$$
y^{\prime \prime}+f_{1}(t) y^{\prime}+f_{0}(t) y=0,
$$

there is a set of transformation that reduce this equation to the form ([6])

$$
y^{\prime}=P(t) y+Q(t) z, z^{\prime}=R(t) y+S(t) z,
$$

where $z(t)$ is an additional unknown function. In the simplest case $P(t) \equiv 0, Q(t) \equiv 1$ we obtain the described above transformation (3).

All these transformations assume that along with the unknown function $y(t),(N-1)$ additional functions are introduced.

There is another kind of transformation that consists in presentation of the solution $y(t)$ of the equation (1) or its derivatives as the sum of the $N$ new unknown functions multiplied by the known functions ${ }^{1}$. By introducing $N$ new unknowns $y_{n}(t)$ instead of the one $y(t)$, we can impose $(N-1)$ additional conditions.

\footnotetext{
${ }^{1}$ Usually, they are the WKB functions.

Such approach can give new form of the $N$-dimensional system of first order equations, equivalent to the equation (1). For the case $N=2$ it is widely used in a theory of electromagnetic waves in stratified media (see, for example, [1, 7-9]). It also was used for developing new solution methods of wave problems from the turning points [10]. In this work we propose some generalization of this transformations ${ }^{2}$ [11].

\section{TRANSFORMATION OF THE $N$-th-ORDER LINEAR DIFFERENTIAL EQUATION}

We represent the solution of the equation (1) as the sum of new functions

$$
y(t)=\sum_{n=1}^{N} y_{n}(t) .
$$

By introducing $N$ new unknowns $y_{n}(t)$ instead of the one $y(t)$, we can impose additional conditions. These conditions we write in the form

$$
\begin{aligned}
& y^{\prime}=\sum_{n=1}^{N} g_{1, n}(t) y_{n}(t), \\
& y^{\prime \prime}=\sum_{n=1}^{N} g_{2, n}(t) y_{n}(t), \\
& \cdots \cdots \\
& y^{(N-1)}=\sum_{n=1}^{N} g_{N-1, n}(t) y_{n}(t),
\end{aligned}
$$

where $g_{m, n}(t)$ are the arbitrary continuous functions having continuous derivatives.

$$
D(t)=\left|\begin{array}{cccc}
1 & 1 & \ldots & 1 \\
g_{1,1}(t) & g_{1,2}(t) & \ldots & g_{1, N}(t) \\
\ldots & \ldots & \ldots & \ldots \\
g_{N-1,1}(t) & g_{N-1,2}(t) & \ldots & g_{N-1, N}(t)
\end{array}\right| \neq 0
$$

then the representation (7)-(8) is unique. Indeed, from (7) and (8) we can uniquely find $y_{n}(t)$ as a linear combination if $y(t)$ and its derivatives. Equations (8) can be rewritten as

\footnotetext{
${ }^{2}$ For the case $N=2$ there are some other transformations of the equation (5) (see, for example, [4]).
} 


$$
\begin{aligned}
& y^{\prime}=\sum_{n=1}^{N} y_{n}^{\prime}(t)=\sum_{n=1}^{N} g_{1, n}(t) y_{n}(t), \\
& y^{\prime \prime}=\left(y_{n}^{\prime}\right)^{\prime}=\sum_{n=1}^{N} g_{1, n}^{\prime}(t) y_{n}(t)+ \\
& +\sum_{n=1}^{N} g_{1, n}(t) y_{n}^{\prime}(t)=\sum_{n=1}^{N} g_{2, n}(t) y_{n}(t), \\
& y^{\prime \prime \prime}=\left(y^{\prime \prime}\right)^{\prime}=\sum_{n=1}^{N} g_{2, n}^{\prime}(t) y_{n}(t)+ \\
& +\sum_{n=1}^{N} g_{2, n}(t) y_{n}^{\prime}(t)=\sum_{n=1}^{N} g_{3, n}(t) y_{n}(t), \\
& \ldots \ldots \\
& y^{(N-2)}=\left(y^{(N-3)}\right)^{\prime}=\sum_{n=1}^{N} g_{N-3, n}^{\prime}(t) y_{n}(t)+ \\
& +\sum_{n=1}^{N} g_{N-3, n}(t) y_{n}^{\prime}(t)=\sum_{n=1}^{N} g_{N-2, n}(t) y_{n}(t), \\
& y^{(N-1)}=\left(y^{(N-2)}\right)^{\prime}=\sum_{n=1}^{N} g_{N-2, n}^{\prime}(t) y_{n}(t)+ \\
& +\sum_{n=1}^{N} g_{N-2, n}(t) y_{n}^{\prime}(t)=\sum_{n=1}^{N} g_{N-1, n}(t) y_{n}(t) . \\
& y^{\prime}
\end{aligned}
$$

The final system of the first order difference equations has the form

$$
\begin{aligned}
& \sum_{n=1}^{N} y_{n}^{\prime}=\sum_{n=1}^{N} g_{1, n} y_{n}, \\
& \sum_{n=1}^{N} g_{m, n} y_{n}^{\prime}=\sum_{n=1}^{N}\left(g_{m+1, n}-g_{m, n}^{\prime}\right) y_{n}, \quad 1 \leq m \leq N-2, \\
& \sum_{n=1}^{N} g_{N-1, n} y^{\prime}=\sum_{n=1}^{N} L_{n} y_{n}-f(t),
\end{aligned}
$$

where

$$
L_{n}=-g_{N-1, n}^{\prime}-f_{N-1} g_{N-1, n}-f_{N-2} g_{N-2, n}-\ldots-f_{0}(t) .
$$

In matrix form

$$
Y^{\prime}(t)=M^{-1} F Y+M^{-1} H,
$$

where $Y=\left(y_{1}, y_{2}, \ldots, y_{N}\right)^{T}, H=(0,0 \ldots,-f(t))^{T}$,

$$
\begin{aligned}
& M=\left(\begin{array}{cccc}
1 & 1 & \ldots & 1 \\
g_{1,1} & g_{1,2} & \ldots & g_{1, N} \\
\ldots & \ldots & \ldots & \ldots \\
g_{N-2,1} & g_{N-2,2} & \ldots & g_{N-2, N} \\
g_{N-1,1} & g_{N-1,2} & \ldots & g_{N-1, N}
\end{array}\right), \\
& F=\left(\begin{array}{cccc}
g_{1,1} & g_{1,2} & \ldots & g_{1, N} \\
g_{2,1}-g_{1,1}^{\prime} & g_{2,2}-g_{1,2}^{\prime} & \ldots & g_{2, N}-g_{1, N}^{\prime} \\
\ldots & \ldots & \ldots & . . \\
g_{N-1,1}-g_{N-2,1}^{\prime} & g_{N-1,2}-g_{N-2,2}^{\prime} & \ldots & g_{N-1, N}-g_{N-2, N}^{\prime} \\
L_{1} & L_{2} & \ldots & L_{N}
\end{array}\right) .
\end{aligned}
$$

We would like to emphasize that $g_{m, n}(t)$ are arbitrary functions that fulfill the condition (9), and we do not demand that these functions separately are the solutions of the equation (1), but their sum must be the solution of this equation.

\section{TRANSFORMATION OF THE SECOND- ORDER LINEAR DIFFERENTIAL EQUATION}

Represent the solution of the equation

$$
y^{\prime \prime}+f_{1}(t) y^{\prime}+f_{0}(t) y+f(t)=0
$$

as the sum of two new functions

$$
y(t)=y_{1}(t)+y_{2}(t) .
$$

Additional condition we write in the form

$$
y^{\prime}=g_{1}(t) y_{1}(t)+g_{2}(t) y_{2}(t) .
$$

Making transformations that are given in section 2 we can write such system of equations

$$
\begin{aligned}
& y_{1}^{\prime}=y_{1} g_{1}-y_{1} \frac{x_{1}}{\left(g_{1}-g_{2}\right)}-y_{2} \frac{x_{2}}{\left(g_{1}-g_{2}\right)}-\frac{f}{\left(g_{1}-g_{2}\right)}, \\
& y_{2}^{\prime}=y_{2} g_{2}+y_{1} \frac{x_{1}}{\left(g_{1}-g_{2}\right)}+y_{2} \frac{x_{2}}{\left(g_{1}-g_{2}\right)}+\frac{f}{\left(g_{1}-g_{2}\right)},
\end{aligned}
$$

where

$$
\begin{aligned}
& x_{1}=g_{1}^{\prime}+f_{1} g_{1}+f_{0}+g_{1}^{2}, \\
& x_{2}=g_{2}^{\prime}+f_{1} g_{2}+f_{0}+g_{2}^{2} .
\end{aligned}
$$

The system (18) is the basis of the proposed transformation in the case of the second-order linear differential equation (15).

Let's consider several cases when special choosing of $g_{1}(t)$ and $g_{2}(t)$ gives useful results.

If we choose

$$
g_{1,2}=\rho_{1,2}(t),
$$

where $\rho_{1,2}$ are the solutions of the characteristic equation

$$
\rho^{2}+f_{1}(t) \rho+f_{0}(t)=0
$$

then the system (18) takes the form

$$
\begin{aligned}
& y_{1}^{\prime}=y_{1} \rho_{1}-y_{1} \frac{\rho_{1}^{\prime}}{\left(\rho_{1}-\rho_{2}\right)}-y_{2} \frac{\rho_{2}^{\prime}}{\left(\rho_{1}-\rho_{2}\right)}-\frac{f}{\left(\rho_{1}-\rho_{2}\right)}, \\
& y_{2}^{\prime}=y_{2} \rho_{2}+y_{1} \frac{\rho_{1}^{\prime}}{\left(\rho_{1}-\rho_{2}\right)}+y_{2} \frac{\rho_{2}^{\prime}}{\left(\rho_{1}-\rho_{2}\right)}+\frac{f}{\left(\rho_{1}-\rho_{2}\right)} .
\end{aligned}
$$

For the case $f_{1}(t) \equiv 0, f(t) \equiv 0$

$$
\begin{aligned}
& y_{1}^{\prime}=\left(i \sqrt{f_{0}}-\frac{f_{0}^{\prime}}{4 f_{0}}\right) y_{1}+y_{2} \frac{f_{0}^{\prime}}{4 f_{0}}, \\
& y_{2}^{\prime}=-\left(i \sqrt{f_{0}}+\frac{f_{0}^{\prime}}{4 f_{0}}\right) y_{2}+y_{1} \frac{f_{0}^{\prime}}{4 f_{0}} .
\end{aligned}
$$

This is the well-known system that is a basis for a coupled wave model and WKB approximation [2, 3, 12 - 16]. We would like to note again that $y_{1}(t)$ and $y_{2}(t)$ separately are not the solutions of equation (15).

Neglecting the second terms on the right-hand side of the equations (23), we obtain the well known WKB result

$$
y_{1,2}=y_{1,2}\left(t_{1}\right)\left(\frac{f_{0}\left(t_{1}\right)}{f_{0}(t)}\right)^{1 / 4} \exp \left( \pm i \int_{t_{1}}^{t} \sqrt{f_{0}} d t^{\prime}\right) .
$$

If $g_{1}(t)$ and $g_{2}(t)$ are the two different solutions of the Riccati equation $[17,18]$

$$
g_{1,2}^{\prime}+f_{1} g_{1,2}+f_{0}+g_{1,2}^{2}=0,
$$

the system (18) transforms into 


$$
\begin{aligned}
& y_{1}^{\prime}=g_{1} y_{1}-\frac{f}{g_{1}-g_{2}}, \\
& y_{2}^{\prime}=g_{2} y_{2}+\frac{f}{g_{1}-g_{2}} .
\end{aligned}
$$

For the homogeneous equation $(f(t) \equiv 0)$

$$
y_{1,2}=y_{1,2}\left(t_{1}\right) \exp \left(\int_{t_{1}}^{t} g_{1,2} d t^{\prime}\right) \text {. }
$$

The derivative of the Wronskian of these functions equals

$$
\begin{gathered}
W^{\prime}=y_{1}\left(t_{1}\right) y_{2}\left(t_{1}\right) \times \\
\times\left[\left(g_{2}-g_{1}\right)^{\prime}+\left(g_{2}^{2}-g_{1}^{2}\right)\right] \exp \left(\int_{t_{1}}^{t}\left(g_{1}+g_{2}\right) d t^{\prime}\right) .
\end{gathered}
$$

Inserting the derivatives of $g_{1,2}$ from (25) into this expression, we obtain

$$
W^{\prime}=-f_{1}(t) W,
$$

that proves the linearly independence of the functions $y_{1,2}$.

From the Riccati equations (25) we can find that $\left(g_{1}(t) \neq g_{2}(t)\right)$

$$
\frac{1}{g_{1}-g_{2}}\left(g_{1}-g_{2}\right)^{\prime}=-\left(g_{1}+g_{2}\right)-f_{1} \text {. }
$$

Denoting the difference of functions $g_{1}$ and $g_{2}$ as

$$
\left(g_{1}-g_{2}\right)=2 i q
$$

then from (30) and (31) we obtain

$$
g_{1,2}= \pm i q-\frac{1}{2 q} q^{\prime}-\frac{f_{1}}{2} .
$$

The solutions (27) can be rewritten in the form that is commonly used in the phase-integral method $[19,20]$

$$
y_{1,2}=y_{1,2}\left(t_{1}\right)\left(\frac{q\left(t_{1}\right)}{q(t)}\right)^{1 / 2} \exp \left( \pm i \int_{t_{1}}^{t} q d t^{\prime}-\frac{1}{2} \int_{t_{1}}^{t} f_{1} d t^{\prime}\right) \text {. }
$$

Inserting (32) into the Riccati equations (25), we find the equation for the $q$ function

$$
\begin{gathered}
g_{1,2}^{\prime}+f_{1} g_{1,2}+f_{0}+g_{1,2}^{2}= \\
=-\frac{1}{2 q} q^{\prime \prime}+\frac{3}{4 q^{2}}\left(q^{\prime}\right)^{2}+f_{0}-q^{2}-\frac{f_{1}^{\prime}}{2}-\frac{f_{1}^{2}}{4}=0,
\end{gathered}
$$

which for $f_{1}(t) \equiv 0$ takes the well-known form [20]

$$
q^{-3 / 2}\left(q^{-1 / 2}\right)^{\prime \prime}+\frac{f_{0}}{q^{2}}-1=0 .
$$

If $g_{1}(t)$ and $g_{2}(t)$ are the solutions of the system of nonlinear differential equations

$$
\begin{aligned}
& g_{1}^{\prime}+f_{1} g_{1}+f_{0}+g_{2} g_{1}=0, \\
& g_{2}^{\prime}+f_{1} g_{2}+f_{0}+g_{2} g_{1}=0,
\end{aligned}
$$

then the system (18) transforms into the "strong" coupling system

$$
\begin{aligned}
& y_{1}^{\prime}=y_{2} g_{2}-\frac{f}{\left(g_{1}-g_{2}\right)}, \\
& y_{2}^{\prime}=y_{1} g_{1}+\frac{f}{\left(g_{1}-g_{2}\right)} .
\end{aligned}
$$

The system (36) can be transformed into the Riccati equation

$$
g_{1}^{\prime}+\left[f_{1}-C \exp \left(-\int_{t_{t}}^{t} f_{1} d t^{\prime}\right)\right] g_{1}+f_{0}+g_{1}^{2}=0
$$

and the relationship between $g_{1}$ and $g_{2}$ is

$$
g_{1}-g_{2}=C \exp \left(-\int_{t_{t}}^{t} f_{1} d t^{\prime}\right)
$$

$C \neq 0$ is the arbitrary constant.

\section{TRANSFORMATION OF THE THIRD- ORDER LINEAR DIFFERENTIAL EQUATION}

Following the section 2, we represent the solution of the third-order linear differential equation (results of detail study of its solutions see in [21])

$$
y^{\prime \prime \prime}+f_{2}(t) y^{\prime \prime}+f_{1}(t) y^{\prime}+f_{0}(t) y+f(t)=0
$$

as the sum of three new functions

$$
y(t)=y_{1}(t)+y_{2}(t)+y_{3}(t) .
$$

Additional conditions we write in the form

$$
\begin{aligned}
& y^{\prime}=g_{1,1}(t) y_{1}(t)+g_{1,2}(t) y_{2}(t)+g_{1,3}(t) y_{3}(t), \\
& y^{\prime \prime}=g_{2,1}(t) y_{1}(t)+g_{2,2}(t) y_{2}(t)+g_{2,3}(t) y_{3}(t) .
\end{aligned}
$$

Applying transformations that are given in section 2, we obtain a system of the first-order linear differential equations

$$
\begin{aligned}
& D y_{1}^{\prime}=y_{1} g_{1,1} D-f\left(g_{1,3}-g_{1,2}\right) \times \\
& \times y_{1}\left[\left(g_{2,2}-g_{2,3}\right)\left(x_{1}-\left(g_{1,1}\right)^{2}\right)-\left(g_{1,3}-g_{1,2}\right)\left(x_{4}+g_{1,1} g_{2,1}\right)\right]+ \\
& +y_{2}\left[\left(g_{2,2}-g_{2,3}\right)\left(x_{2}-\left(g_{1,2}\right)^{2}\right)-\left(g_{1,3}-g_{1,2}\right)\left(x_{5}+g_{1,2} g_{2,2}\right)\right]+ \\
& +y_{3}\left[\left(g_{2,2}-g_{2,3}\right)\left(x_{3}-\left(g_{1,3}\right)^{2}\right)-\left(g_{1,3}-g_{1,2}\right)\left(x_{6}+g_{1,3} g_{2,3}\right)\right], \\
& D y_{2}^{\prime}=y_{2} g_{1,2} D-f\left(g_{1,1}-g_{1,3}\right) \times \\
& \times y_{1}\left[\left(g_{2,3}-g_{2,1}\right)\left(x_{1}-\left(g_{1,1}\right)^{2}\right)-\left(g_{1,1}-g_{1,3}\right)\left(x_{4}+g_{1,1} g_{2,1}\right)\right]+ \\
& +y_{2}\left[\left(g_{2,3}-g_{2,1}\right)\left(x_{2}-\left(g_{1,2}\right)^{2}\right)-\left(g_{1,1}-g_{1,3}\right)\left(x_{5}+g_{1,2} g_{2,2}\right)\right]+ \\
& +y_{3}\left[\left(g_{2,3}-g_{2,1}\right)\left(x_{3}-\left(g_{1,3}\right)^{2}\right)-\left(g_{1,1}-g_{1,3}\right)\left(x_{6}+g_{1,3} g_{2,3}\right)\right], \\
& D y_{3}^{\prime}=y_{3} g_{1,3} D-f\left(g_{1,2}-g_{1,1}\right) \times \\
& \times y_{1}\left[\left(g_{2,1}-g_{2,2}\right)\left(x_{1}-\left(g_{1,1}\right)^{2}\right)-\left(g_{1,2}-g_{1,1}\right)\left(x_{4}+g_{1,1} g_{2,1}\right)\right]+ \\
& +y_{2}\left[\left(g_{2,1}-g_{2,2}\right)\left(x_{2}-\left(g_{1,2}\right)^{2}\right)-\left(g_{1,2}-g_{1,1}\right)\left(x_{5}+g_{1,2} g_{2,2}\right)\right]+ \\
& +y_{3}\left[\left(g_{2,1}-g_{2,2}\right)\left(x_{3}-\left(g_{1,3}\right)^{2}\right)-\left(g_{1,2}-g_{1,1}\right)\left(x_{6}+g_{1,3} g_{2,3}\right)\right],
\end{aligned}
$$

where the following notations were introduced

$$
\begin{aligned}
& x_{1}=\left(g_{2,1}-g_{1,1}^{\prime}\right), \\
& x_{2}=\left(g_{2,2}-g_{1,2}^{\prime}\right), \\
& x_{3}=\left(g_{2,3}-g_{1,3}^{\prime}\right), \\
& x_{4}=\left(g_{2,1}^{\prime}+f_{2} g_{2,1}+f_{1} g_{1,1}+f_{0}\right), \\
& x_{5}=\left(g_{2,2}^{\prime}+f_{2} g_{2,2}+f_{1} g_{1,2}+f_{0}\right), \\
& x_{6}=\left(g_{2,3}^{\prime}+f_{2} g_{2,3}+f_{1} g_{1,3}+f_{0}\right) .
\end{aligned}
$$

If we choose 


$$
\begin{aligned}
& g_{1, n}=\rho_{n}, \\
& g_{2, n}=\rho_{n}^{2}, n=1,2,3,
\end{aligned}
$$

where $\rho_{n}$ are the solutions of the equation

$$
\rho^{3}+f_{2}(t) \rho^{2}+f_{1}(t) \rho+f_{0}(t)=0,
$$

then the system (45) takes the form

$$
\begin{aligned}
& y_{1}^{\prime}=y_{1} \rho_{1}-y_{1} \rho_{1}^{\prime}\left(\frac{1}{\rho_{1}-\rho_{3}}+\frac{1}{\rho_{1}-\rho_{2}}\right)-y_{2} \rho_{2}^{\prime}\left(\frac{1}{\rho_{1}-\rho_{2}}+\frac{1}{\rho_{3}-\rho_{1}}\right)- \\
& -y_{3} \rho_{3}^{\prime}\left(\frac{1}{\rho_{1}-\rho_{3}}+\frac{1}{\rho_{2}-\rho_{1}}\right)-f \frac{\rho_{3}-\rho_{2}}{D}, \\
& y_{2}^{\prime}=y_{2} \rho_{2}-y_{1} \rho_{1}^{\prime}\left(\frac{1}{\rho_{2}-\rho_{2}}+\frac{1}{\rho_{3}-\rho_{2}}\right)-y_{2} \rho_{2}^{\prime}\left(\frac{1}{\rho_{2}-\rho_{1}}+\frac{1}{\rho_{2}-\rho_{3}}\right)- \\
& -y_{3} \rho_{3}^{\prime}\left(\frac{1}{\rho_{1}-\rho_{3}}+\frac{1}{\rho_{2}-\rho_{1}}\right)-f \frac{\rho_{1}-\rho_{3}}{D}, \\
& y_{3}^{\prime}=y_{3} \rho_{3}-y_{1} \rho_{1}^{\prime}\left(\frac{1}{\rho_{3}-\rho_{3}}+\frac{1}{\rho_{2}-\rho_{3}}\right)-y_{2} \rho_{2}^{\prime}\left(\frac{1}{\rho_{3}-\rho_{2}}+\frac{1}{\rho_{1}-\rho_{3}}\right)- \\
& -y_{3} \rho_{3}^{\prime}\left(\frac{1}{\rho_{3}-\rho_{1}}+\frac{1}{\rho_{3}-\rho_{3}}\right)-f \frac{\rho_{2}-\rho_{1}}{D},
\end{aligned}
$$

where $D=\left(\rho_{3}-\rho_{1}\right)\left(\rho_{3}-\rho_{2}\right)\left(\rho_{2}-\rho_{1}\right)$. This system coincides with the one that was obtained in [2] by another method. In the WKB approximation

$$
\begin{aligned}
& y_{1}^{\prime}=y_{1} \rho_{1}-y_{1} \rho_{1}^{\prime}\left(\frac{1}{\rho_{1}-\rho_{3}}+\frac{1}{\rho_{1}-\rho_{2}}\right)-f \frac{\rho_{3}-\rho_{2}}{D}, \\
& y_{2}^{\prime}=y_{2} \rho_{2}-y_{2} \rho_{2}^{\prime}\left(\frac{1}{\rho_{2}-\rho_{1}}+\frac{1}{\rho_{2}-\rho_{3}}\right)-f \frac{\rho_{1}-\rho_{3}}{D}, \\
& y_{3}^{\prime}=y_{3} \rho_{3}-y_{3} \rho_{3}^{\prime}\left(\frac{1}{\rho_{3}-\rho_{1}}+\frac{1}{\rho_{3}-\rho_{3}}\right)-f \frac{\rho_{2}-\rho_{1}}{D} .
\end{aligned}
$$

These equations have the solutions which coincide with results of asymptotic analysis (see, for example, [4]).

If we choose the functions $g_{m, n}$ that are the solutions of the following equations

$$
\begin{aligned}
& x_{1}=\left(g_{2,1}-g_{1,1}^{\prime}\right)=g_{1,1}^{2}, \\
& x_{2}=\left(g_{2,2}-g_{1,2}^{\prime}\right)=g_{1,2}^{2}, \\
& x_{3}=\left(g_{2,3}-g_{1,3}^{\prime}\right)=g_{1,3}^{2}, \\
& x_{4}=\left(g_{2,1}^{\prime}+f_{2} g_{2,1}+f_{1} g_{1,1}+f_{0}\right)=-g_{1,1} g_{2,1}, \\
& x_{5}=\left(g_{2,2}^{\prime}+f_{2} g_{2,2}+f_{1} g_{1,2}+f_{0}\right)=-g_{1,2} g_{2,2}, \\
& x_{6}=\left(g_{2,3}^{\prime}+f_{2} g_{2,3}+f_{1} g_{1,3}+f_{0}\right)=-g_{1,3} g_{2,3},
\end{aligned}
$$

the system (45) takes the form

$$
\begin{aligned}
& y_{1}^{\prime}=y_{1} g_{1,1}-f \frac{g_{1,3}-g_{1,2}}{D}, \\
& y_{2}^{\prime}=y_{1} g_{1,2}-f \frac{g_{1,1}-g_{1,3}}{D}, \\
& y_{3}^{\prime}=y_{3} g_{1,3}-f \frac{g_{1,2}-g_{1,2}}{D} .
\end{aligned}
$$

From (51) it follows that functions $g_{m, n}$ are the three different solutions of the system of the first-order nonlinear differential equations

$$
\begin{aligned}
& g_{1, n}^{\prime}+g_{1, n}^{2}-g_{2, n}=0, \\
& g_{2, n}^{\prime}+f_{2} g_{2, n}+f_{1} g_{1, n}+f_{0}+g_{1, n} g_{2, n}=0 .
\end{aligned}
$$

This system can be written as the second-order nonlinear differential equation $g_{1, n}^{\prime \prime}+g_{1, n}^{\prime}\left(3 g_{1, n}+f_{2}\right)+f_{1} g_{1, n}+f_{0}+f_{2} g_{1, n}^{2}+g_{1, n}^{3}=0$

For the simplest case $f_{2} \equiv 0, f_{1} \equiv 0$,

$$
g_{1, n}^{\prime \prime}+g_{1, n}^{\prime} 3 g_{1, n}+g_{1, n}^{3}+f_{0}=0 .
$$

In contrast to the Duffing equation (see, for example, [22]), the equation (55) has no linear term proportional to $g_{1, n}$.

For the third-order linear equation the equation (54) (or system (53)) plays the same role as the Riccati equation for second-order linear equation.

The functions $y_{n}=\exp \left(\int_{t_{1}}^{t} g_{1, n} d t^{\prime}\right)$ are linear independent, and the general solution of the homogeneous equation $(40)(f \equiv 0)$ is

$$
y(t)=\sum_{n=1}^{3} y_{n}\left(t_{1}\right) \exp \left(\int_{t_{1}}^{t} g_{1, n} d t^{\prime}\right) .
$$

There are other forms of system of the first order equations that can be obtained from the system (45) by choosing functions $g_{m, n}$.

\section{CONCLUSIONS}

We presented a transformation of the linear differential equation into a system of the first order equations. The proposed transformation gives possibility to get new forms of the $N$-dimensional system of the first order equations that can be useful for analysis of the solutions of the $N$ th order differential equation. In particular, for the third-order linear equation the nonlinear second-order equation that plays the same role as the Riccati equation for the second-order linear equation is obtained. The new form of the system of first-order equations can also be used for finding WKB solutions of the linear difference equation with coefficients that vary sufficiently slowly with index.

\section{REFERENCES}

1. K.G. Budden. Radio waves in the ionosphere. Cambridge University Press, 1961.

2. J. Heading. The Nonsingular Embedding of Transition Processes within a More General Framework of Coupled Variables // Journal of Research of the $\mathrm{Na}$ tional Bureau of Standards-D. Radio Propagation. 1961, v. 65D, № 6, p. 595-616.

3. K.G. Budden, M.S. Smith. Phase Memory and Additional Memory in W.K.B. Solutions for Wave Propagation in Stratified Media // Proc. R. Soc. Lond. A. 1976, v. 350, p. 27-46.

4. M.V. Fedoryuk. Asymptotic Analysis. Linear Ordinary Differential Equations. Springer, 1993.

5. M. Bologna. Exact analytical approach to differential equations with variable coefficients // Eur. Phys. J. Plus. 2016, 131:386; E-print arxiv:1505.06509v1, 2015.

6. E. Kamke. Differentialgleichungen Lösungsmethoden und Lösungen Springer Fachmedien. Wiesbaden, 1977.

7. L.M. Brekhovskikh. Waves in Layered Media. Academic Press, 1960. 
8. J.R. Wait. Electromagnetic Waves in Stratified Media. Pergamon Press, 1970.

9. M.I. Rabinovich, D.I. Trubetskov. Oscillations and Waves in Linear and Nonlinear Systems. Springer, 1989.

10. N.E. Tsapenko. A general method for solving a second-order homogeneous linear equation // Differ. Uravn. 1979, v. 15, № 6, p. 1050-1053.

11. M.I. Ayzatsky. A note on the transformation of the linear differential equation into a system of the first order equations // E-print arxiv.org/abs/1803.03124, 2018.

12. W.H. Louisell. Coupled mode and Parametric Electronics. Wiley, 1960.

13. A.H. Haus, H.W. Weiping. Coupled-Mode Theory. // Proceedings of the IEEE. 1991, v. 19, № 10, p. $1505-1518$

14. A.A. Barybin, V.A. Dmitriev. Modern Electrodynamics and Coupled-mode Theory: Application to Guided-wave Optics. Rinton Press, 2002.
15. T.S.L. Radhika, T.K.V. Iyengar, T. Raja Rani. Approximate Analytical Methods for Solving Ordinary Differential Equations. CRC Press, 2014.

16. C.M. Bender, S.A. Orszag. Advanced Mathematical Methods for Scientists and Engineers. Springer, 1978.

17. W.T. Reid. Riccati Differential Equations. Academic Press, 1972.

18. A.I. Egorov. Riccaty Equation. Pensoft Publishers, 2007.

19. J. Heading. An Introduction to Phase-Integral Methods. Dover Publications, 2003.

20. N. Fröman, P.O. Fröman. Physical Problem solved by the phase-integral method. Cambridge UP, 2004.

21. M. Gregus. Third Order Linear Differential Equations. Springer, 1987.

22. I. Kovacic, M.J. Brennan (Eds.). The Duffing Equation. Wiley, 2011.

Article received 28.03.2019

\section{О ТРАНСФОРМАЦИИ ЛИНЕЙНОГО ДИФФЕРЕНЦИАЛЬНОГО УРАВНЕНИЯ В СИСТЕМУ РАЗНОСТНЫХ УРАВНЕНИЙ ПЕРВОГО ПОРЯДКА}

\section{Н.И. Айзацкий}

Представлено обобщение преобразования линейного дифференциального уравнения в систему разностных уравнений первого порядка. Предложенное преобразование дает возможность получить новые формы $\mathrm{N}$-мерной системы уравнений первого порядка, которые могут быть полезны для анализа решений дифференциальных уравнений третьего порядка. В частности, для линейного дифференциального уравнения третьего порядка получено нелинейное дифференциальное уравнение второго порядка, которое играет ту же роль, что и уравнение Риккати для линейного дифференциального уравнения второго порядка.

\section{ПРО ТРАНСФОРМАЦІЮ ЛІНІЙНОГО ДИФЕРЕНЦІАЛЬНОГО РІВНЯННЯ В СИСТЕМУ РІЗНИЦЕВИХ РІВНЯНЬ ПЕРШОГО ПОРЯДКУ}

\section{M.I. Айзацький}

Представлено узагальнення перетворення лінійного диференціального рівняння в систему різницевих рівнянь першого порядку. Запропонована трансформація дає можливість отримати нові форми $N$-вимірної системи рівнянь першого порядку, які можуть бути корисними для аналізу розв'язків диференціальних рівнянь. Зокрема, для лінійного диференціального рівняння третього порядку отримано нелінійне рівняння другого порядку, яке відіграє ту ж саму роль, що і рівняння Ріккаті для лінійного рівняння другого порядку. 